\title{
DALLA GIURISDIZIONE DELLA CORTE SUPREMA ALLA COMPETENZA DEL TRIBUNALE SPECIALE: LA SFIDA DEL SISTEMA GIUDIZIARIO ALBANESE CONTRO LA CORRUZIONE AD ALTO LIVELLO GOVERNATIVO
}

\author{
Ismail Tafani* \\ * Former Lecturer at Albanian University (Albania), now Lecturer at Barleti University (Albania). \\ (ismailtafani@yahoo.it)
}

\begin{abstract}
The Constitution of the Republic of Albania, adopted in 1998, provided that the Supreme Court had primary and cassation jurisdiction. Primary jurisdiction referred to the obligation attributed to this Court to judge at first instance, the most important figures of the political and judicial power. Although there is a perception of the corruption of the subjects of this jurisdiction, which was an exception to the right of the second instance court of law, the Supreme Court had not shown itself to be independent in the judicial processes of high-level officials. In 2016, when the constitutional reform of the judicial system in Albania was adopted, the provision that created hope for the fight against corruption at high levels of government was the establishment of the Special Tribunal against corruption and organized crime and the Special Investigative Structure (SPAK). The latter is required by the constitutional reform to independently investigate senior state officials, unlike what the Attorney General had done before the reform. The implementation of the reform, in this respect, is ongoing, however, yet has not been presented an outcome of the judicial reform, but what deserves attention is an analysis of the expectations of the Special Court, unlike the jurisdiction of the first instance of the Supreme Court. The question that arises is whether this constitutional change will transform the approach of the judicial system or will it remain yet another reform written on paper. The doubts, in this context, arise from the fact that five years have passed since the reform started and the challenge of defeating corruption and strengthening the rule of law still remains an illusion that it is expected to turn into delusion.
\end{abstract}

Keywords: Corruption, Immunity, Impunity, Special Court, Special Structure, Supreme Court Jurisdiction. 


\title{
NGA JURISDIKSIONI I GJYKATËS SË LARTË TEK KOMPETENCA E GJYKATËS SË POSAÇME: SFIDA E SISTEMIT GJYQËSOR SHQIPTAR KUNDËR KORRUPSIONIT TË LARTË QEVERITAR
}

\author{
Ismail Tafani* $^{*}$ \\ * Ish-pedagog në Albanian University (Shqipëri), tani Pedagog në Universitetin Barleti (Shqipëri). \\ (ismailtafani@yahoo.it)
}

\begin{abstract}
Abstrakt
Kushtetuta e Republikës së Shqipërisë, e miratuar në vitin 1998, parashikonte që Gjykata e Lartë të kishte juridiksion parësor dhe atë të kasacionit. Juridiksioni parësor i referohej detyrimit që $i$ atribuohet kësaj Gjykate për të gjykuar në shkallë të parë figurat më të rëndësishme të pushtetit politik dhe gjyqësor. Edhe pse ekziston një perceptim për korrupsionin e subjekteve të këtij juridiksioni, që përbënte përjashtim nga e drejta e gjykatës së shkallës së dytë, Gjykata e Lartë nuk ishte treguar e pavarur në proceset gjyqësore të zyrtarëve të lartë. Në vitin 2016, kur u miratua reforma kushtetuese e sistemit gjyqësor në Shqipëri, dispozita që krijoi shpresë për luftën kundër korrupsionit në nivelet e larta të qeverisjes ishte ngritja e Gjykatës së Posaçme për Korrupsionin dhe Krimin e Organizuar (GJKKO) dhe Struktura e Posaçme Hetimore (SPAK). Kësaj të fundit $i$ kërkohet nga reforma kushtetuese që të hetojë në mënyrë të pavarur zyrtarët e lartë të shtetit, ndryshe nga sa kishte bërë Prokurori i Përgjithshëm para reformës. Zbatimi $i$ reformës, në këtë aspekt, është në vazhdim, por ende nuk është paraqitur një rezultat $i$ reformës në drejtësi, por ajo që meriton vëmendje është analiza e pritshmërive të Gjykatës Speciale, ndryshe nga juridiksioni fillestar i Gjykatës së Lartë. Pyetja që shtrohet është nëse ky ndryshim kushtetues do të transformojë qasjen e sistemit gjyqësor apo do të mbetet një reformë tjetër e shkruar në letër. Dyshimet, në këtë kontekst, lindin nga fakti se kanë kaluar pesë vjet nga fillimi i reformës dhe sfida e mposhtjes së korrupsionit dhe forcimit të shtetit ligjor mbetet ende një iluzion që pritet të kthehet në zhgënjim.
\end{abstract}

Fjalë kyçe: korrupsioni, imuniteti, pandëshkueshmëria, Gjykata e Posaçme, Strukturë e Posaçme, jurisdiksioni i Gjykatës së Lartë. 


\section{Considerazioni generali sulle riforme costituzionali in Albania per sconfiggere la corruzione dei rappresentanti dei poteri dello Stato}

Le sfide dell'Albania per quanto riguarda il funzionamento e l'effettività degli organi statali nell'implementazione della legislazione sembrano essere infinite. Queste sfide, di volta in volta, si sovrappongono nel raggiungere gli obiettivi della società e dello Stato albanese. In questo senso, diventa necessario sottolineare che la sfida principale, la quale merita un'elaborazione particolare, è uno dei principi cardine della Costituzione, ovvero quello dell'uguaglianza. La sfida del sistema giudiziario albanese è di rendere la legge uguale per tutti e far sì che la legge non perdoni in nessuna parte delle sue disposizioni, anche quando si tratta di persone con un'immunità costituzionale oppure politica.

Per quanto riguarda le nuove istituzioni del sistema giudiziario albanese, riformato dalla riforma costituzionale del 2016, verrà condotta di seguito, in questo studio, un'analisi comparativa con il sistema precedente, senza pretendere di esaurire tutti gli argomenti. Si tratta della sfida di sconfiggere la corruzione e la criminalità organizzata, fenomeni che sembrano di avere "infettato" i livelli più alti governativi e che paradossalmente sembrano di stare in simbiosi l'uno con l'altro. La convivenza della corruzione ad alti livelli governativi con la criminalità organizzata, oltre ad essere un pericolo per il consolidamento del principio dello Stato di diritto, rappresenta nello stesso tempo il vero problema per lo Stato e la società albanese nel lungo e difficile processo di diventare parte della "famiglia" dell'Unione Europea.

La "sconfitta" del sistema giudiziario albanese nei confronti della corruzione ad alti livelli governativi e della criminalità organizzata aveva portato alla perdita di fiducia della società in questo sistema e nelle istituzioni che la rappresentavano. Questa pare sia stata la ragione alla base e il punto di partenza della riforma costituzionale sul sistema giudiziario albanese. La riforma costituzionale di questo sistema ha previsto diversi approcci nei confronti dei rappresentanti dei tre poteri dello stato per il rafforzamento delle istituzioni costituzionali.

Per i giudici di tutti i gradi, compresi quelli della Corte costituzionale, dal 2017 è iniziato ed è ancora in corso il cosiddetto processo di vetting, previsto per Costituzione e che si sta realizzando in base alla legge (1). Questo processo, come era previsto dalla riforma costituzionale, ha creato delle istituzioni ad hoc che hanno il potere di operare un controllo tridimensionale dei giudici nonché dei pubblici ministeri. Invece, per i rappresentanti politici del popolo, cioè quelli che rappresentano gli altri due poteri dello Stato, ad oltre cinque anni dalla riforma costituzionale, non si vedono dei risultati significativi. I risultati significativi, in questo senso, sono quelli che, tramite un sistema giudiziario indipendente, farebbero perdere ai politici, quell'immunità che li vede immuni anche nei confronti del principio dello Stato di diritto.

La riforma costituzionale del sistema giudiziario albanese ha preso l'ispirazione dalla perdita di fiducia della società in questo sistema nonché dai rapporti della Commissione europea che evidenziavano una necessità di riforma (2). Dunque, la ragione principale della riforma sembra che sia stata condizionata dalla richiesta degli Stati Uniti e dell'Unione Europea per la continuazione del processo d'integrazione dell'Albania in quest'ultima (3). 
Nel 2015, cioè un anno prima della riforma costituzionale del sistema giudiziario albanese, è stata intrapresa una riforma costituzionale per impedire alle persone con un passato criminale di candidarsi alle elezioni parlamentari e locali e a coprire altre cariche di alto livello governativo (4). Questa riforma ha preceduto la riforma del sistema giudiziario albanese e ha previsto l'approvazione della legge d'attuazione con una maggioranza qualificata per garantire l'integrità dei candidati, degli eletti e quelli nominati ad alti livelli governativi (5). Per l'attuazione di questa riforma serviva altresì una decisione del parlamento albanese (6) che ha avuto come obbiettivo di facilitare il processo tramite $\mathrm{i}$ formulari di autodichiarazione che i soggetti coinvolti nella legge dovevano compilare.

Insomma, queste riforme costituzionali, l'una dopo l'altra, sembrano aver preso atto dell'alto livello di corruzione tra i più alti rappresentanti dei poteri dello Stato. Pare inevitabile la necessità di "purificare" tutti i poteri dello Stato in Albania, poiché, non rispettando più il principio della separazione dei poteri, avevano iniziato a perdere la funzione loro assegnata dalla Costituzione. Dunque, non è azzardato dire che da poteri dello Stato erano diventati poteri contro lo Stato.

Dopo il processo di vetting dei giudici e dei pubblici ministeri, in Albania si è parlato anche di un processo di vetting dei politici, ma non si è raggiunto il consenso politico necessario ad approvare una riforma del genere. Ovviamente, quest'approccio anche per i politici avrebbe trattato in egual modo i rappresentanti di tutti i poteri dello Stato. In questo modo, la lotta alla corruzione sembrerebbe seria, poiché questo fenomeno era previsto come reato in Albania, ancora prima che questa diventasse uno Stato, cioè a partire dalle prime norme consuetudinarie (7).

Il vetting dei giudici e dei pubblici ministeri, come si è detto, si basava sul controllo tridimensionale, cioè del patrimonio, della figura e delle capacità professionali, ovviamente avendo come base il controllo patrimoniale (8). Seguendo la logica di un processo efficiente nell'evidenziare la corruzione, probabilmente un controllo del patrimonio dei politici avrebbe dato degli effetti soddisfacenti nella stessa maniera dei giudici e pubblici ministeri, perlomeno ad allontanarli perché i processi penali necessitano di istituzioni più consolidate.

Invece, il legislatore albanese ha preferito istituire un Tribunale Speciale per combattere la corruzione ad alti livelli governativi e quindi non ha ritenuto necessario un vetting dei politici, nonostante la società albanese abbia mostrato di avere perso la fiducia nei confronti del potere politico, cioè negli altri due poteri dello Stato, quindi legislativo ed esecutivo, altrettanto quanto nel potere giudiziario.

Dal 2015, le grandi e profondi riforme costituzionali in Albania hanno avuto un unico obiettivo, ovvero crescere la fiducia nelle istituzioni dello Stato albanese da parte della società albanese, degli Stati Uniti e dell'Unione europea. Una fiducia che potrebbe realizzarsi solo con la lotta alla corruzione e alla criminalità organizzata, ma, a quanto pare, realizzata in diversi modi per i diversi poteri dello Stato. Comunque sia, queste riforme fanno capire che il pluralismo politico in Albania aveva fallito nella formazione di una governabilità dello Stato, nel rispetto del principio costituzionale della separazione dei poteri. 
Le due vie perseguite per portare alla funzionalità le istituzioni ad oggi hanno dato dei risultati diversi al fine combattere la parte "infettata" dei poteri. Per quanto riguarda il vetting, che ha avuto un meccanismo simile ad un processo amministrativo, risulta che siano stati rimossi dall'ufficio tanti giudici e pubblici ministeri. Pur essendo rimossi dall'ufficio perché non hanno potuto giustificare il patrimonio che possiedono, nulla è stato fatto a proposito dell'apertura di procedimenti penali nei loro confronti.

Invece, per quanto riguarda i poteri politici, si attende il funzionamento del Tribunale Speciale anticorruzione e criminalità organizzata e della nuova Struttura Speciale Investigativa (SPAK), ma in questo caso tramite un processo penale, poiché il processo penale per gli eventuali reati degli alti funzionari dello Stato, prima della riforma, era di competenza della Corte Suprema. Quest'ultima era senza dubbio sottomessa al potere politico dello Stato, il quale aveva fatto si che perdesse totalmente l'indipendenza principio necessario per avere dei processi giudiziari e, di conseguenza, delle sentenze imparziali, nel rispetto del principio dello Stato di diritto -.

\section{La Corte Suprema albanese nel giudizio di prima istanza da un'eccezione del diritto del doppio grado di giudizio ad una garanzia dell'impunibilità}

L'articolo 141 della Costituzione albanese del 1998 prevedeva che la Corte Suprema avesse giurisdizione primaria nei confronti delle più alte cariche dello Stato. La sua giurisdizione primaria si riferiva ai procedimenti penali nei confronti dei rappresentanti delle istituzioni costituzionali, partendo dal Presidente della Repubblica fino ai giudici della stessa Corte Suprema (9). Evidentemente, il costituente albanese aveva previsto una garanzia superiore nei confronti delle figure più importanti dello Stato, cioè il loro eventuale processo penale solo dalla Corte suprema, però a discapito del diritto al doppio grado di giudizio.

In tal senso, pare ovvio specificare che la previsione dell'articolo 43 (10) della Costituzione albanese nella versione del 1998 (e, in particolare, la previsione del comma 2 dello stesso) - che sanciva il diritto del doppio grado di giudizio tranne l'eccezione che si prevede al primo coma dell'articolo 141 - "non sembra prefiguri possibilità di filtri nell'accesso alla giurisdizione della Corte, ma solo meccanismi di distribuzione interna" (11).

L'articolo 136 comma 1, della Costituzione albanese prima della riforma costituzionale del sistema giudiziario del 2016, sanciva che "I componenti della Corte Suprema sono nominati dal Presidente della Repubblica su consenso dell'Assemblea parlamentare”. La formazione della Corte Suprema in questo modo, prima del 2016, con la volontà dei poteri politici ha reso vana la sua giurisdizione di prima istanza ai sensi dell'articolo 141, nei confronti di coloro che la formavana ai sensi dell'articolo 136 primo comma, come sopra citato. L'esercizio dell'azione penale nei confronti delle alte cariche dello Stato era di competenza dell'ufficio della Procura Generale come previsto dall'articolo 13 della Legge n. 8737 del 2001 (12). In più, allo stesso modo, era pregiudicato anche l'esercizio dell'azione penale, se si ha riguardo anche al comma 1 dell'articolo 149 della Costituzione, che prevedeva la nomina del Procuratore Generale con la seguente formulazione: "Il Procuratore Generale è nominato dal Presidente della Repubblica su 
consenso dell'Assemblea parlamentare per un mandato quinquennale con diritto di rinomina.". Per quanto riguarda i processi penali, ai sensi dell'articolo 141, 1 comma, i risultati si comprendono facilmente dal punto di vista teorico, ma trovano conferma anche nella prassi, la quale ha creato il cosiddetto "principio d'impunibilità".

In tal senso, nessuna delle istituzioni, né la Procura Generale o la Corte suprema, ha mostrato di essere indipendente, come per Costituzione doveva essere, mostrando quasi sempre un timore nei confronti dei poteri politici. In effetti, la maggior parte delle cause portate in giudizio alla Corte suprema sono state a querela di parte. Tuttavia, in quei pochi procedimenti penali avviati d'ufficio dalla Procura Generale, le sentenze sono state sempre di proscioglimento. I procedimenti penali di prima istanza davanti alla Corte suprema hanno avuto come oggetto prevalente i reati di ingiuria e di diffamazione. Ai sensi del Codice di procedura penale albanese, questi reati sono perseguibili su querela di parte, con la sola partecipazione del Procuratore competente. Inoltre, bisogna specificare che da quando è stato modificato il Codice penale albanese nel 2012 sono punibili solo con la pena pecuniaria (13).

Di contro, con la riforma costituzionale del 2016, 1'articolo 43 della Costituzione albanese è stato modificato come segue: "Ogni persona ha il diritto di proporre ricorso contro una decisione del Tribunale, ad un Tribunale superiore, salvo che la legge disponga diversamente per i delitti di lieve entità, per le materie civili o amministrative di minore importanza o valore, alle condizioni previste dall'articolo 17 della Costituzione". In effetti, l'articolo 17 della Costituzione Albanese prevede una sorta di confine all'eccezioni dei diritti in essa garantiti, che sono quelli previsti dalla Convenzione europea per la salvaguardia dei diritti dell'uomo e delle libertà fondamentali (CEDU).

Per quanto riguarda il diritto al doppio grado di giudizio, la CEDU sancisce questo diritto, nel quadro del processo penale, all'articolo 2 del Protocollo n. 7 (14), rubricato "Diritto a un doppio grado di giudizio in materia penale". Come si vede, la Costituzione albanese ha fatto proprio quanto statuito dalla CEDU a proposito dell'eccezioni al diritto del doppio grado di giudizio, che ogni Stato parte può prevedere per legge. La riforma costituzionale albanese, adeguandosi alla CEDU, passa da un'eccezione del diritto al doppio grado di giudizio, sancito dalla Costituzione ante-riforma, a una previsione come letteralmente prevista dalla CEDU, fondantesi perciò su una riserva di legge.

La Costituzione albanese non prevedeva un doppio grado di giudizio per i processi penali contro le più alte cariche dello Stato, se non il ricorso alla Corte costituzionale, come era previsto per tutti gli individui. Questo ricorso si legittimava, in caso di violazione del diritto al giusto processo, sulla scorta di quanto previsto dell'articolo 6 della CEDU e dall'articolo 42 della Costituzione albanese. La riforma del sistema giudiziario in questo senso ha esteso il ricorso individuale alla Corte Costituzionale per la protezione di tutti i diritti previsti dalla Costituzione, ovviamente dopo aver esaurito tutti i gradi di giudizio. La previsione costituzionale dell'articolo 141 della Costituzione albanese sulla giurisdizione di prima istanza della Corte suprema, sanciva ovviamente una garanzia per quei soggetti che, qualora diversamente prevista, poteva portare a risultati opposti. Ciò vale a dire che avrebbe creato una magistratura forte che avrebbe sbilanciato quegli equilibri necessari sottesi al principio della separazione dei poteri. La previsione dell'unico grado di giudizio della Corte suprema nel processo penale per le alte cariche 
dello Stato - che a loro volta nominavano i suoi giudici - aveva fortemente minato l'indipendenza della Corte. La conseguenza di questa scelta del costituente nel 1998 è stata, oltre alla perdita di fiducia nei confronti del potere giudiziario, anche la creazione del cosiddetto "principio d'impunibilità" nei confronti dei rappresentanti dei poteri politici.

Tuttavia, lo standard previsto dalla Costituzione albanese per limitare il diritto al doppio grado di giudizio era evidenziato dalla Corte costituzionale come uno standard che superava le previsioni della CEDU (15). Questo standard dell'eccezione del diritto del doppio grado di giudizio pare avesse condotto ad una sottomissione del potere giudiziario agli altri due poteri dello Stato. La sottomissione del potere giudiziario, oltre ad essere servito come una minaccia a quegli equilibri necessari nell'implementazione del principio di separazione dei poteri, aveva finito per aumentare la percezione della società che la corruzione significasse potere, anziché l'inverso.

Atteso che la Corte suprema si formasse per volontà politica, tale circostanza pare fosse diventata parte della politica in Albania (16) e, a volte, anche causa del conflitto tra i diversi partiti che a loro volta volevano dominarla totalmente. Da questo punto di vista, appare compressibile la sua sottomissione agli altri poteri dello Stato e, di conseguenza, il suo "fallimento" nella lotta alla corruzione ad alti livelli governativi.

\section{La Struttura Speciale anticorruzione e la competenza del Tribunale Speciale come una speranza per sconfiggere il "principio d'impunibilità" in Albania}

È oramai risaputo che la riforma costituzionale del sistema giudiziario in Albania ha avuto come prima intenzione quella di riportare la fiducia della popolazione al sistema stesso, ma è altrettanto risaputo l'altro obiettivo che, allo stesso tempo, è il suo nucleo. In questa logica, non si può tralasciare la creazione del Tribunale Speciale anticorruzione e criminalità organizzata. Questo Tribunale dovrebbe sostituire la giurisdizione di prima istanza della Corte suprema, allargando l'alveo dei soggetti, come era previsto nel primo comma dell'articolo 141 della Costituzione. Teoricamente, la riforma costituzionale del 2016 ha previsto la creazione di un Tribunale Speciale che sia indipendente e, di conseguenza, imparziale nei processi penali a carico di politici corrotti, fino ad oggi "intoccabili" dalla "rete" del sistema giudiziario. La causa di tale "intoccabilità" era da ascriversi alla trasformazione del principio costituzionale della separazione dei poteri, come previsto dalla Costituzione del 1998, in "principio" della condivisione e collaborazione tra i potenti, che altro non erano che i rappresentanti dei tre poteri dello Stato.

Si potrebbe dire che dal nuovo Tribunale Speciale e dalla nuova Struttura Speciale anticorruzione e criminalità organizzata ci si attenda una vera e propria rivoluzione nello sradicare il concetto che riguarda l'impunibilità delle alte cariche dello Stato in Albania. Queste aspettative si riferiscono alla società albanese, al fine di (ri)creare la fiducia nelle istituzioni, ma soprattutto alla comunità internazionale (e qui si vuole intendere, in particolare, gli Stati Uniti e l'UE, quali veri promotori della riforma costituzionale). Se si parla di corruzione in Albania, non si trovano ragioni per non collegare fortemente questo fenomeno con la politica. Anzi, per la società albanese sembra che le parole siano persino sinonimi. Più potere si ha, più alta è la probabilità che se ne abusi e, anche se quest'abuso equivale a reato, alla fine tutto dipende dal procedimento penale. Quando quest'ultimo 
manca, l'abuso del potere è solo una percezione che svanisce con il passare del tempo, a discapito del principio dello Stato di diritto.

La riforma costituzionale del 2016 - che fu approvata con la legge di revisione costituzionale - è riuscita ad avere il sostegno di tutti i 140 parlamentari del Parlamento albanese, mostrando di essere la riforma più voluta dall'approvazione della Costituzione nel 1998. La Legge di revisione costituzionale n. 76/2016 ha previsto all'articolo 13 - che ha modificato l'articolo 135 della Costituzione - l'istituzione del Tribunale Speciale che diventa competente rationae materiae nei procedimenti penali contro la corruzione e la criminalità organizzata e, allo stesso tempo, nei procedimenti penali contro le alte cariche dello Stato (17).

L'istituzione del Tribunale Speciale per combattere la corruzione di tutte le alte cariche dello Stato, come evidenziato nella Costituzione, dimostra che esiste una forte percezione di essere governati da persone corrotte $\mathrm{e}$, a volte, collegate con la criminalità organizzata, come dimostra anche il diffuso dibattito pubblico sul punto in Albania. L'istituzione dell'Alto Consiglio della Magistratura (18), a composizione maggioritaria degli stessi giudici, pone delle garanzie per la creazione di una magistratura indipendente, compresa quella della Corte Suprema. Ovviamente, quest'ultima non avrà più il ruolo di giudice di prima istanza, ma comunque è parte del sistema giudiziario.

Presso il Tribunale Speciale anticorruzione è prevista l'istituzione della Procura Speciale, dotata di un Ufficio di polizia giudiziaria ad hoc per iniziare i procedimenti, come previsto dalla Costituzione. Le modifiche costituzionali hanno reso necessaria la modifica del Codice di procedura penale, ove viene dettagliata la competenza del Tribunale Speciale. Così, l'articolo 75/a del codice di Procedura Penale albanese è stato modificato, specificando la competenza del Tribunale Speciale anticorruzione e criminalità organizzata (19). Tale Tribunale giudica i reati di corruzione e i reati di criminalità organizzata in tutte le forme previste dal Codice Penale albanese, nonché i reati commessi dai soggetti elencati all' articolo 135 della Costituzione. I reati dei soggetti quali gli alti funzionari possono essere di ogni natura e sono di competenza per materia dal Tribunale Speciale. Il nome di questo Tribunale e la logica della riforma costituzionale del sistema giudiziario rendono chiaro l'obiettivo di quest'istituzione, che è appunto la lotta alla corruzione ad alti livelli governativi.

Una delle novità importanti della riforma costituzionale del 2016 è stata la creazione di un organo collegiale per garantire l'indipendenza della Procura e, di conseguenza, l'esercizio dell'azione penale. Il nuovo organo collegiale si chiama "Alto Consiglio della Procura" e dovrebbe garantire l'indipendenza dei pubblici ministeri (20). Questa previsione crea le basi di un autogoverno dei pubblici ministeri, visto che la maggioranza dell'Alto Consiglio della Procura è composta da questi, che si eleggono in seno alla propria associazione. Da questo punto di vista, la riforma ha un approccio totalmente diverso da quanto previsto dal sistema previgente, quando l'esercizio dell'azione penale dipendeva dal Procuratore Generale, vista la centralizzazione dell'organizzazione della Procura, come analizzato anche dalla Corte Suprema in Sezioni Unite (21).

Inoltre, sempre in funzione dell'esercizio dell'azione penale nei confronti dei soggetti contemplati all'articolo 135 della Costituzione - come modificato dalla riforma costituzionale del 2016 -, l'articolo 148/dh, stabilisce il ruolo e la composizione della Procura Speciale, nonché il controllo che deve essere operato su tali soggetti e sul Nucleo Speciale Investigativo e loro familiari, in modo che questi non abusino della loro indipendenza durante l'esercizio dell'azione penale (22). 
Poiché la procura è stata decentralizzata come forma di organizzazione, i conflitti di competenza tra la Procura ordinaria e la Procura anticorruzione si risolvono con la decisione del Capo Procuratore della Procura Speciale, come modificato nel Codice di Procedura Penale albanese nel 2017. Così, l'articolo 89 del Codice di Procedura Penale prevede che la Procura Speciale anticorruzione e criminalità organizzata, in base alla sua valutazione, ha la precedenza per materia nei casi di conflitto con la Procura presso i tribunali ordinari.

Dopo la riforma costituzionale è nata la necessità di approvare diverse leggi a fini attuativi, che prevedessero una trasformazione della governabilità all'interno del potere giudiziario. L'obiettivo è quello d'impedire agli altri poteri dello Stato di porre in discussione l'indipendenza del potere giudiziario. Tuttavia, rendere indipendente il potere giudiziario in Albania probabilmente richiederà tempo, nonostante le nuove istituzioni di autogoverno della magistratura e della Procura avessero individuato tale tempo in otto mesi dall'entrata in vigore della riforma.

Tra le leggi nuove vi è anche la legge organica che regola il funzionamento della Struttura Speciale anticorruzione e criminalità organizzata. La Struttura Speciale comprende la Procura Speciale e il Nucleo Speciale Investigativo (23), nonché la polizia giudiziaria, ricadenti sotto l'abbreviazione SPAK, come previsto dalla stessa legge. La legge che prevede la regolarizzazione della nuova struttura anticorruzione è la n. 95/2016 sull'organizzazione e sul funzionamento delle istituzioni contro la corruzione e la criminalità organizzata (24).

L'implementazione di questa legge ha richiesto molto tempo per dare forza all'azione penale e far sì che il nucleo della riforma costituzionale - sia concesso esprimersi metaforicamente in tal senso - "disinfestasse" lo stato albanese dalla corruzione. L'indipendenza della polizia giudiziaria - dotata di un suo Direttore, il cui processo di nomina è stato alquanto lungo, giacché si è passati attraverso la verifica dell'integrità personale tramite la macchina della verità e dei corsi di addestramento svolti negli Stati Uniti e in Italia, presso la Procura Antimafia Italiana - dipenderà solo dalla Procura Speciale.

Tuttavia, ad oggi dalla riforma costituzionale del sistema giudiziario albanese non ne sono derivati risultati concreti, atti a sradicare il cosiddetto "principio d'impunibilità" delle persone che coprono rilevanti cariche costituzionali, oltre le altre previste per legge. Nonostante le aspettative, ad oltre cinque anni dalla riforma probabilmente è ancora presto per fare un'analisi completa, dato che ci si trova all'inizio della sua implementazione. Comunque sia, non è troppo sottolineare una frase che fa parte del pluralismo politico in Albania ed è ciò che riguarda la non implementazione della legge, che sembra sia il più grande problema del funzionamento dello Stato di diritto in Albania.

\section{Conclusioni}

Tramite questo studio, si è analizzato il concetto d'impunibilità dei soggetti che rivestono ruoli importanti nelle istituzioni costituzionali e in quelle previste per legge in Albania. In buona sostanza, per combattere la corruzione in Albania e per cambiare la percezione della società verso quelli che la governano, si è reso necessario processar alti funzionari dello Stato soprattutto politici. La Costituzione albanese del 1998 aveva previsto un unico grado di giudizio per i reati delle alte cariche dello Stato. La composizione della Corte Suprema albanese determinata dall'intervento della volontà politica con il passare degli 
anni aveva fatto perdere la sua indipendenza nei confronti degli altri poteri dello Stato. Per quanto riguarda la giurisdizione di prima istanza della Corte Suprema, si conclude che era diventata un "ostaggio" del potere politico, che aveva un ruolo significativo nella sua formazione.

Risulta dallo studio che, nonostante la Corte Suprema avesse svolto diversi procedimenti giudiziari di natura penale di prima istanza, la maggior parte dei reati che venivano contestati ai soggetti di elevato ruolo politico era su querela di parte e non il risultato dell'esercizio ex officio dell'azione penale dell'ufficio della Procura Generale per reati di corruzione. Dunque, il ruolo della Procura Generale a iniziare d'ufficio procedimenti penali per questi soggetti, cioè per reati più gravi, era pressoché inesistente. La maggior parte delle sentenze della Corte Suprema nei giudizi di prima istanza erano sentenze di proscioglimento oppure di condanna con la pena pecuniaria, senza intervenire - neppure lontanamente - sulla percezione popolare del fenomeno corruttivo, che sembra "fiorire" ad alti livelli governativi.

Si conclude, inoltre, che la creazione di un Tribunale Speciale contro la corruzione e la criminalità organizzata - che deve giudicare i politici corrotti - può dare speranza a battere il cosiddetto "principio d'impunibilità", creato durante gli anni del pluralismo politico in Albania. Ovviamente, questa previsione costituzionale - che conferisce ad un Tribunale Speciale contro la corruzione e la criminalità organizzata la competenza per materia dei reati commessi da soggetti importanti e dotati di immunità costituzionale e politica - prende atto dell'inefficienza della Corte Suprema per i giudizi di prima istanza. Per il funzionamento dello Stato è necessario che i suoi poteri siano bilanciati, al fine di rendere vivo il principio costituzionale della separazione dei poteri e, quindi, rendere la legge uguale per tutti. Inoltre, questa previsione costituzionale prevede la realizzazione del diritto del doppio grado di giudizio anche per le alte cariche dello Stato, che verranno processate laddove nei loro confronti venga imputato un qualunque reato dal Tribunale Speciale contro la corruzione e criminalità organizzata, con la possibilità di fare appello al Tribunale Speciale d'Appello e ricorso alla Corte Suprema, come previsto dalla legge. Per ultimo, si conclude che, come in ogni riforma in Albania, a maggior ragione per questa - che vuole riconfermare la forza dello Stato di diritto -, il divario dall'approvazione all'implementazione è come un "grande mare". Tuttavia, spetta al tempo dimostrare che le nuove istituzioni, istituite dalla riforma costituzionale del sistema giudiziario albanese 2016, possano battere una volta per sempre il cosiddetto "principio d'impunibilità" in Albania e riuscire a implementare il principe cardine della giustizia, cioè quello per cui la legge è uguale per tutti.

\section{References}

(1) Legge n. 84/2016, "Sulla rivalutazione transitoria di giudici e pubblici ministeri nella Repubblica d'Albania".

(2) Commissione speciale parlamentare per la riforma del sistema di giustizia, Gruppo di esperti di alto livello, Analisi del sistema giudiziario in Albania, Giugno 2015, disponibile al link http://www.reformanedrejtesi.al/sites/default/files/dokumenti_shqip_0.pdf, p. 10 ss.

(3) I. TAFANI, Constitutional Reform of the Justice System in Albania "Condicio sine qua non" for Country's Integration into the European Union, 4th International Conference, "Problems and Challenges of the transformation of the Albanian Society Towards the Standards of the European Union", organizzato dall' Albanian University, 22 dicembre 2017, Tirana, Albania. 
(4) Legge n. 137/2015 "Su alcune modifiche ed integrazioni alla Legge n. 8417, Data 21.10.1998, Costituzione della Repubblica d'Albania" e successive modifiche.

(5) Legge n. 138/2015 "Sulla garanzia dell'integrità delle persone elette, nominate o esercitanti funzioni pubbliche".

(6) Decisione n. 17/2016 del Parlamento albanese per la determinazione delle modalità di attuazione dei divieti previsti dalla Legge n. 138/2015 "Sulla garanzia dell'integrità delle persone elette, nominate o esercitanti funzioni pubbliche".

(7) E. CUKANI, A mali estremi, estremi rimedi: maladministration e misure anti-corruzione in Albania, in DPCE Online, [S.1.], Vol. 38, No. 1, 2019, disponibile al link http://www.dpceonline.it/index.php/dpceonline/article/view/669, p. 324.

(8) Legge n. 84/2016, cit. V. S. HASANI, L'Albania al bivio di un compromesso: la riforma della giustizia albanese e l'apertura dei negoziati con l'Unione europea, in AIC-Osservatorio Costituzionale, Fasc. 3, 2020.

(9) Legge n. 8417, del 21 ottobre 1998, "Costituzione della Repubblica d'Albania", e successive modifiche agli articoli: 43, 141, 147, 148/dh e 149. L'articolo 141: "1. La Corte Suprema ha giurisdizione primaria e di cassazione. Essa ha giurisdizione primaria quando giudica le accuse penali contro il Presidente della Repubblica, contro il Presidente e i componenti del Consiglio dei Ministri, i deputati, i giudici della Corte Suprema e contro i giudici della Corte costituzionale. 2. Per l'uniformità o per la modifica della giurisprudenza, la Corte Suprema ha diritto di richiamare ad esaminare a sezioni unite determinate questioni".

(10) Legge n. 8417, del 21.10.1998, "Costituzione della Repubblica d'Albania", l'articolo 43 "Ognuno ha diritto di ricorrere in appello con una sentenza a una corte superiore tranne nei casi previsti dalla Costituzione"

(11) S. BENVENUTI, La riforma del sistema giudizario albanese riflessioni su Corte Suprema e consiglio di giustizia in ottica comparata, in Nomos: Le attualità nel diritto, Fasc. 2, 2015, p. 3.

(12) Legge n. 8737 del 12 febbraio 2001 "Sull'organizzazione e il funzionamento della Procura nella Repubblica d'Albania" e successive modifiche.

(13) Legge n. 23/2012 "Su alcune integrazioni e modifiche alla legge n. 7895", del 27.1.1995, Codice Penale della Repubblica d'Albania", e successive modifiche.

(14) Convenzione Europea dei Diritti dell'Uomo, come modificata dai Protocolli nn. 11, 14 e 15, Protocolli nn. 1, 4, 6, 7, 12, 13 e 16. L'art. 2 del Protocollo n. 7 sancisce "1. Ogni persona dichiarata colpevole da un tribunale ha il diritto di far esaminare la dichiarazione di colpevolezza o la condanna da una giurisdizione superiore. L'esercizio di tale diritto, ivi compresi i motivi per cui esso può essere esercitato, è disciplinato dalla legge. 2. Tale diritto può essere oggetto di eccezioni per reati minori, quali sono definiti dalla legge, o quando l'interessato è stato giudicato in prima istanza da un tribunale della giurisdizione più elevata o è stato dichiarato colpevole e condannato a seguito di un ricorso avverso il suo proscioglimento".

(15) Corte costituzionale albanese, sentenza del 05 luglio 2005, n. 14. In questa sentenza, la Corte Costituzionale chiarisce la posizione di questo diritto costituzionale in comparazione con la previsione della CEDU.

(16) A. VORPSI, A. ANASTASI, G. IBRAHIMI, S. BERBERI, S. SADUSHI (eds.), Komentar. Mbi reformën kushtetuese në sistemin e drejtësisë (2016), Tiranë, 2019, pp. 203-204.

(17) Legge n. 76/2016, del 22 luglio 2016, "Per alcune integrazioni e modifiche alla legge n. 8417, del 21.10.1998, Costituzione della Repubblica d'Albania", e successive modifiche. Il comma 2 dell'articolo 13 ridisegna in modo radicale l'articolo 135 della Costituzione albanese con la formulazione "(...)2. I tribunali speciali giudicano i reati di corruzione e criminalità organizzata, nonché i reati contro il Presidente della Repubblica, del Presidente dell'Assemblea, del Presidente del Consiglio dei ministri, del membro del Consiglio dei ministri, del giudice della Corte costituzionale e della Suprema Corte, del Procuratore Generale, del l'Alto Ispettore di Giustizia, del Sindaco, del parlamentare, del vice Ministro, del membro dell'Alto Consiglio Giudiziario e dell'Alto Consiglio della Procura, e dei Capi delle istituzioni centrali o indipendenti come definite dalla Costituzione o dalla legge, nonché le accuse contro gli ex funzionari, predetti".

(18) Legge n. 8417, del 21 ottobre 1998, cit. L'articolo 147 commi 1 e 2 della Costituzione albanese "1. L'Alto Consiglio della Magistratura garantisce l'indipendenza, la responsabilità e l'andamento della magistratura nella Repubblica d'Albania. 2. L'Alto Consiglio della Magistratura è composto da 11 membri, 
sei dei quali sono eletti da giudici di tutti i gradi della magistratura e cinque membri sono eletti dall'Assemblea Parlamentare, tra i giuristi non giudici".

(19) Legge n.7905, del 21 marzo 1995 "Codice di Procedura Penale della Repubblica d'Albania", come modificato dalla legge n. 35/2017, del 30 marzo 2017.

(20) Legge n. 8417, del 21 ottobre 1998, cit.

(21) Corte Suprema, Sezioni Unite, sentenza del 14 ottobre 2002, n.4 "Funzioni del Pubblico Ministero".

(22) Legge n. 8417, del 21 ottobre 1998, cit. L'articolo 148/dh inserito nella Costituzione albanese dopo la riforma del 2016 "1. La Procura speciale svolge l'azione penale e rappresenta l'accusa davanti ai tribunali speciali previsti dall'articolo 135, comma 2, della Costituzione, nonché davanti alla Corte Suprema. 2. La Procura Speciale è composta da almeno 10 pubblici ministeri, nominati dal Consiglio Superiore della Procura per 9 anni, senza diritto di riconferma. La legge stabilisce altri criteri per la selezione nonché per la procedura di nomina trasparente e pubblica. 3. Il Capo della Procura speciale è eletto tra i procuratori di questa Procura a maggioranza dei membri dell'Alto Consiglio della Procura, per 3 anni, senza diritto di rielezione, a norma di legge. 4. Il Procuratore della Procura Speciale è destituito per aver commesso un reato o per aver commesso una grave violazione disciplinare con due terzi dei membri del Consiglio Superiore della Procura. 5. Il candidato alla carica di pubblico ministero, funzionario incaricato delle indagini, personale amministrativo della Procura speciale, Nucleo speciale investigativo, nonché i suoi stretti familiari, prima della nomina, si sottopongono a verifica patrimoniale e di immagine, prestano il consenso al controllo periodico dei propri conti bancari e delle telecomunicazioni personali, per legge".

(23) Il Nucleo Speciale Investigativo è la polizia giudiziaria di questa strutura, sulla scia del modello statunitense. Si chiama in albanese (Byroja Kombëtare Hetimit) per rendere assomigliante il suo nome con il FBI americano.

(24) Legge n. 95/2016 "Sull'organizzazione e sul funzionamento delle istituzioni contro la corruzione e la criminalità organizzata". 